\title{
Metastable Crystalline and Amorphous Fe-V Alloys Produced by Vapor Quenching
}

\author{
By N. Kataoka*, K. Sumiyama** and Y. Nakamura** \\ $\mathrm{Fe}_{1-x} \mathrm{~V}_{x}$ alloys obtained by vapor quenching have been investigated by $\mathrm{X}$-ray diffraction, magnetiza- \\ tion and Mössbauer effect measurements. The single bcc phase appears for $0 \leqq x \leqq 0.3$ and $0.6<x \leqq 1.0$, \\ while the single amorphous phase for $0.45 \leqq x \leqq 0.5$, where the $\sigma$ phase is stable in the equilibrium phase \\ diagram. Since the bcc and amorphous phases are magnetically distinct, the volume fraction of the \\ paramagnetic amorphous phase was estimated. The Mössbauer spectra indicate that the amorphous \\ alloys have a short range atomic arrangement similar to that of the $\sigma$ phase.
}

(Received June 16, 1986)

Keywords: iron-vanadium alloys, metastable phase, amorphous alloys, sigma phase, sputtering, vapor quenching, Mössbauer effect

\section{Introduction}

Vapor-quenching has been known as a powerful method of producing new metastable alloys. When metal components are deposited onto a cold substrate, the mobility of arriving atoms is greatly reduced and amorphous alloys as well as metastable crystalline alloys can be stabilized in violation of the equilibrium phase diagram $^{(1)}$.

Systematic studies have been made for Fe-based binary alloys produced by vapor quenching ${ }^{(2)-(4)}$. In vapor quenched $\mathrm{Fe}_{1-x} \mathrm{Ti}_{x}$ alloys, the amorphous phase has been produced for $0.2<x<0.75$, where the C14 type Laves phase and the B2 type ordered phase are stable in the equilibrium phase diagram ${ }^{(5)-(7)}$. The amorphous $\mathrm{Fe}_{1-x} \mathrm{Ti}_{x}$ alloys are thermally the most stable for $x=0.3-0.4$, where we have the Laves phase in the equilibrium states $^{(8)}$, i.e., in the vapor quenched $\mathrm{Fe}-\mathrm{Ti}$ alloys the formation of an amorphous phase and its thermal stability are intimately correlated with the Laves phase intermetallic compound. It is important to know whether or not this close relation between an amorphous phase and an

* Graduate Student, Kyoto University, Kyoto 606, Japan.

** Department of Metal Science and Technology, Faculty of Engineering, Kyoto University, Kyoto 606, Japan. intermetallic compound is general. In the equilibrium $\mathrm{Fe}_{1-x} \mathrm{~V}_{x}$ alloys, another intermetallic compound, the $\sigma$ phase, exists over $0.37<x<0.57$ at low temperatures, while an amorphous alloy has been produced by sput tering for $x=0.34^{(4)}$. The structure of the $\sigma$ phase, which is one of the topologically-closepacked (TCP) phases, is tetragonal with 30 atoms per unit cell and the space group $D_{4 h^{-}}^{14}$ $\mathrm{P}_{2} / \mathrm{mnm}$. In the equilibrium phase diagram, a bcc phase is stable for $0 \leqq x<0.23$ and $0.66<x \leqq 1$ at low temperatures. At high temperatures, however, the bcc phase is stable for $0 \leqq x \leqq 1.0^{(9)}$.

This paper shows experimental results of Xray diffraction, magnetization and Mössbauer effect measurements for sputtered $\mathrm{Fe}_{1-x} \mathrm{~V}_{x}$ alloys with $x=0-1.0$, with special attention to the relation between the amorphous alloys and the $\sigma$ phase. On the basis of the Mössbauer experiments, the local environments of the nonequilibrium phases will be compared with those of the equilibrium phases.

\section{Experimental Procedures}

$\mathrm{Fe}-\mathrm{V}$ alloy targets were prepared from 99.9\% $\mathrm{Fe}$ and $99.7 \% \mathrm{~V}$ by arc-melting in an argon atmosphere. Composite type targets were prepared from $99.9 \% \mathrm{Fe}$ and $99.7 \% \mathrm{~V}$ disk plates, where the alloy composition was controlled by the ratio of the surface areas of 
Table 1 Sputtering parameters.

\begin{tabular}{ll}
\hline \hline Input power & $160 \mathrm{~W}$ \\
Background pressure & $<1.1 \times 10^{-5} \mathrm{~Pa}$ \\
& $\left(0.8 \times 10^{-7} \mathrm{Torr}\right)$ \\
& $5.3 \times 10^{-1} \mathrm{~Pa}$ \\
Ar gas pressure & $\left(4 \times 10^{-3} \mathrm{Torr}\right)$ \\
& $\sim 0.13 \mathrm{~nm} / \mathrm{s}(\sim 80 \AA / \mathrm{min})$ \\
Deposition rate & $350 \mathrm{~K}$ \\
Substrate temperature & $2 \sim 3 \mu \mathrm{m}$ \\
Film thickness & polyimide \\
Substrate & \\
\hline \hline
\end{tabular}

Fe and $\mathrm{V}$ metal plates on the target.

A few micron thick alloy films were deposited on a polyimide substrate by a conventional rf sputtering method, whose operational parameters are listed in Table 1. The composition of sputtered alloy films was determined by fluorescence X-ray analysis and EPMA.

$\mathrm{X}$-ray diffraction measurements using $\mathrm{Co}-$ $\mathrm{K} \alpha$ radiations were made at $290 \mathrm{~K}$. The magnetization was measured by a torsion balance magnetometer at temperatures from $4.2 \mathrm{~K}$ to $290 \mathrm{~K}$ in magnetic fields up to $1.3 \mathrm{~T}$. The ${ }^{57} \mathrm{Fe}$ Mössbauer effect measurements were made at $290 \mathrm{~K}$ and $4.2 \mathrm{~K}$ by a constant acceleration type spectrometer using a ${ }^{57} \mathrm{Co}$ source doped in $\mathrm{Cu}$. The velocity scale is relative to $\alpha$ $\mathrm{Fe}$ at $290 \mathrm{~K}$.

\section{Experimental Results}

Figure 1(a) and (b) show low angle parts of $\mathrm{X}$-ray diffraction patterns of the vapor quenched $\mathrm{Fe}_{1-x} \mathrm{~V}_{x}$ alloys. Only a broad bcc (110) diffraction line is observed for $x<0.4$ and $x>0.6$, while a halo pattern is observed for $0.45<x<0.5$. For $0.5<x<0.6$, an extra line is observed on the higher angle side of the bcc (110) line of the disordered alloys, although these lines are not clearly resolved for $x=0.59$. This is identified with a (110) line of the B2 type ordered alloy, because the lattice constant of the B2 type alloy is smaller than that of the disordered bcc alloy ${ }^{(10)}$. In the vapor quenched $\mathrm{Fe}-\mathrm{V}$ alloys, the half widths of diffraction lines are much wider than those of pure $\mathrm{Fe}$ and $\mathrm{V}$ powder specimens due probably to lattice defects, incorporated $\mathrm{Ar}$ atoms and small grain sizes of deposited films.

Figure 2 shows the lattice constants of the

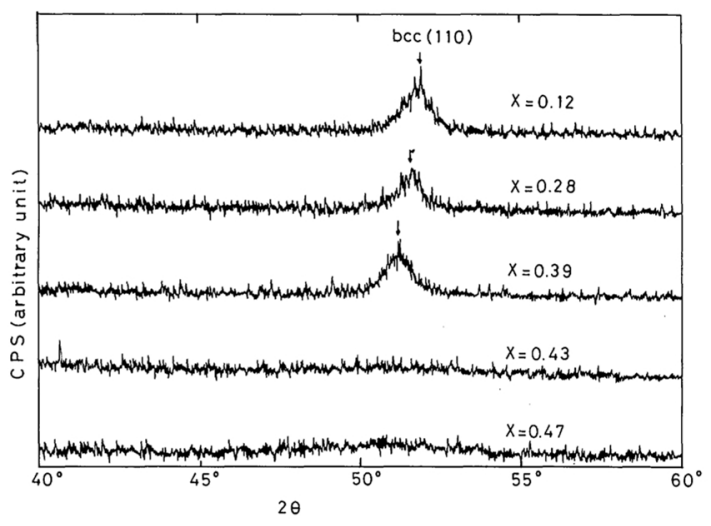

(a)

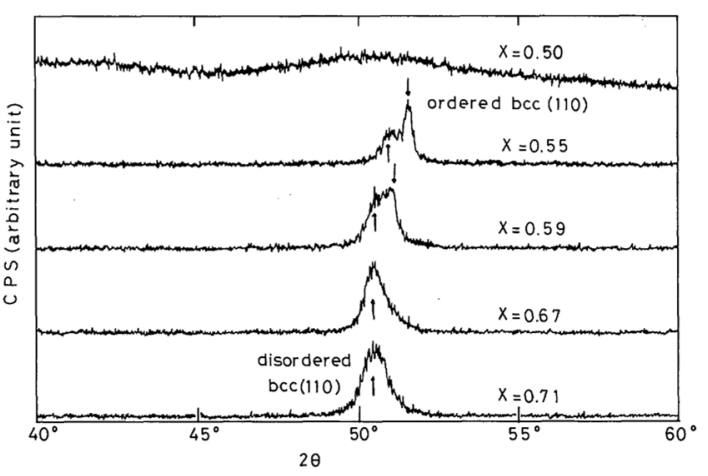

(b)

Fig. 1 Low angle parts of X-ray diffraction patterns of the vapor quenched $\mathrm{Fe}_{1-x} \mathrm{~V}_{x}$ alloys. (a) $0.1<x<0.5$, (b) $0.5 \leqq x<0.8$.

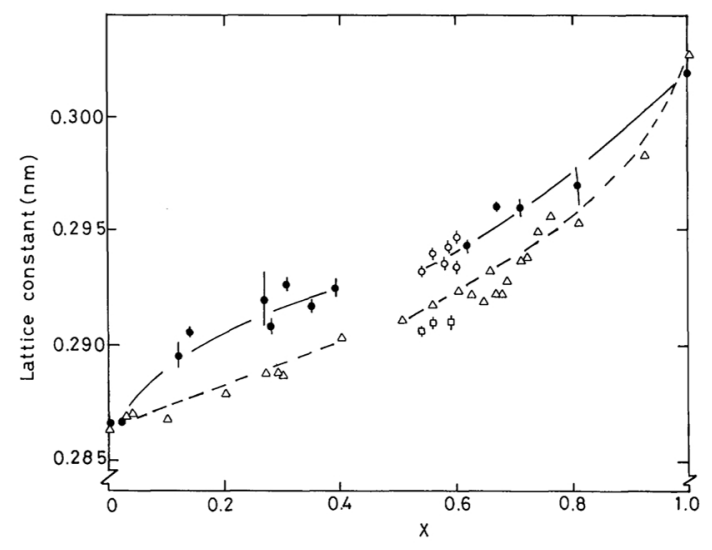

Fig. 2 Lattice constant of the bcc $\mathrm{Fe}_{1-x} \mathrm{~V}_{x}$ alloys at 290 K. $\bigcirc$ : the present vapor quenched alloys. $\triangle$ : the bulk disordered alloys ${ }^{(10)} . \bigcirc$ and $\square$ : the disordered and ordered states of the present vapor quenched alloys in the two-phase region, respectively. 


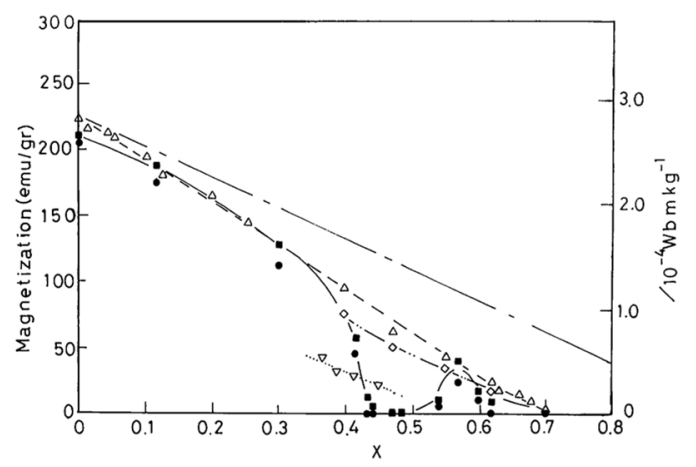

Fig. 3 Magnetization versus $\mathrm{V}$ concentration, $x$, for the $\mathrm{Fe}_{1-x} \mathrm{~V}_{x}$ alloys. $\square$ and $\bullet$ : the present vapor quenched alloys at $4.2 \mathrm{~K}$ and $290 \mathrm{~K}$, respectively. $\triangle$ : the bulk disordered bcc alloys at $4.2 \mathrm{~K}^{(12)} . \diamond$ : the bulk ordered bcc alloys at $4.2 \mathrm{~K}^{(12)} . \nabla$ : the bulk $\sigma$ alloys at $4.2 \mathrm{~K}^{(13)}$. The broken line indicates the Slater-Pauling curve and the dash-dotted line indicates the simple dilution law.

bcc $\mathrm{Fe}_{1-x} \mathrm{~V}_{x}$ alloys estimated from X-ray diffraction measurements. The lattice constant increases with the increase in $x$, being always larger than that of bulk $\mathrm{Fe}-\mathrm{V}$ alloys. This lattice expansion may be ascribed to the incorporation of $\mathrm{Ar}$ atoms and interstitial atoms produced by a peening effect ${ }^{(11)}$.

The concentration dependence of the magnetization of the $\mathrm{Fe}_{1-x} \mathrm{~V}_{x}$ alloys is shown in Fig. 3. The bulk bce and $\sigma$ phases are ferromagnetic at low temperatures ${ }^{(12)(13)}$. The magnetization of vapor quenched bcc $\mathrm{Fe}_{1-x} \mathrm{~V}_{x}$ alloys decreases with increasing $x$ for $x<0.3$, being in agreement with the results of the bulk bcc Fe-V alloys. It rapidly decreases beyond about $x=0.4$, in accordance with the appearance of the amorphous phase. The amorphous $\mathrm{Fe}_{1-x} \mathrm{~V}_{x} \quad(x=0.45-0.5)$ alloys are paramagnetic $^{(4)}$. With further increasing $x$, the magnetization again appears and has a maximum at about $x=0.57$, corresponding to the appearance of the bcc Fe-V phase.

${ }^{57} \mathrm{Fe}$ Mössbauer spectra at $4.2 \mathrm{~K}$ for the vapor quenched $\mathrm{Fe}_{1-x} \mathrm{~V}_{x}$ alloys are shown in Fig. 4. A broad ferromagnetic sextet is observed for $x<0.3$, while a paramagnetic spectrum for $0.4<x<0.6$. With the further increase in $x$, a broad ferromagnetic spectrum is observed because of the appearance of the bcc phase. These results are consistent with the results of X-ray diffraction and magnetization

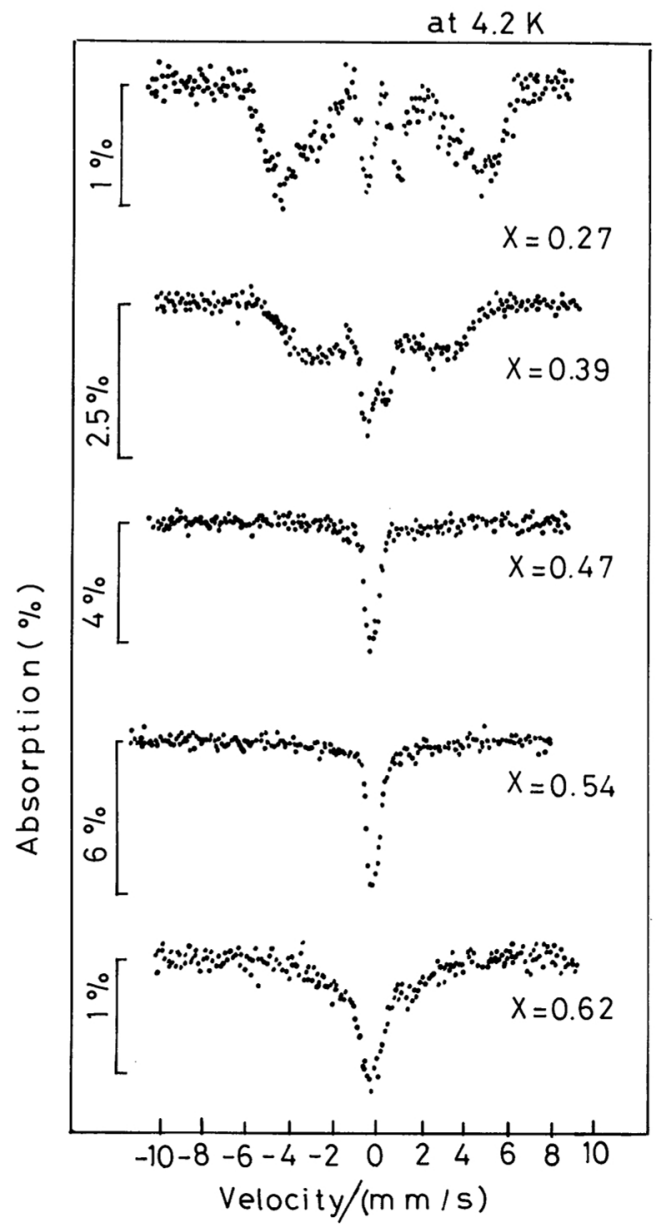

Fig. 4 Mössbauer spectra of the vapor quenched $\mathrm{Fe}_{1-x} \mathrm{~V}_{x}$ alloys at $4.2 \mathrm{~K}$. The velocity scale is relative to $\alpha$-Fe at $290 \mathrm{~K}$.

measurements. Each spectrum was fitted by a superposition of sets of six Lorentzians with different Zeeman splittings by a method of Hesse and Rübartsch ${ }^{(14)}$. In this analysis, the smoothing factor was chosen as 50 . The isomer shift and the intensity ratios of the second and fifth peaks to the others were adjusted to get the best fitting. The contribution of the electric quadrupole interaction was neglected for simplicity. The average hyperfine field, $\bar{H}_{\mathrm{hf}}$, obtained by this method is shown in Fig. 5: the concentration dependence of $\bar{H}_{\mathrm{hf}}$ of the bcc phase is similar to that of the bulk bcc alloys ${ }^{(15)}$ and to the results of magnetization measurements shown in Fig. 3.

The Mössbauer spectra at $290 \mathrm{~K}$ for the 


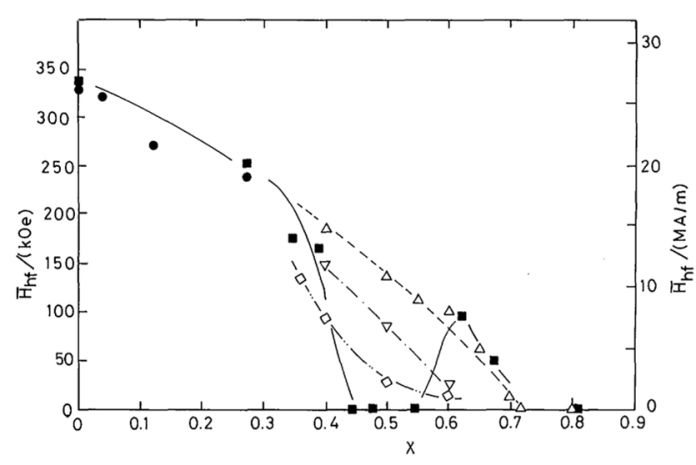

Fig. 5 Average hyperfine field, $\bar{H}_{\text {hf }}$, versus $V$ concentration, $x$, for the $\mathrm{Fe}_{1-x} \mathrm{~V}_{x}$ alloys. $\mathbf{\square}$ and $\boldsymbol{\bullet}$ : the present vapor quenched alloys at $4.2 \mathrm{~K}$ and $290 \mathrm{~K}$, respectively. $\triangle$ : the bulk disordered bcc alloys at $4.2 \mathrm{~K}^{(15)} . \nabla$ : the bulk ordered bcc alloys at $4.2 \mathrm{~K}^{(15)}$. $\diamond$ : the bulk $\sigma$ alloys at $4.2 \mathrm{~K}^{(27)}$.

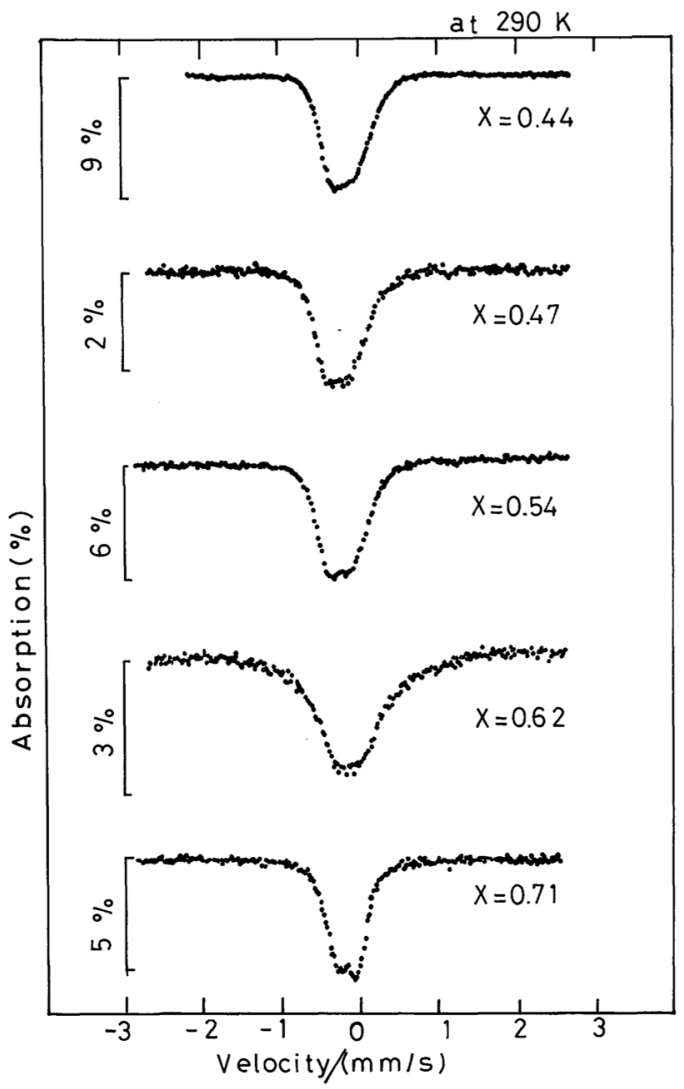

Fig. 6 Mössbauer spectra at $290 \mathrm{~K}$ for the vapor quenched $\mathrm{Fe}_{1-x} \mathrm{~V}_{x}$ alloys. The velocity scale is relative to $\alpha$-Fe at $290 \mathrm{~K}$.
$\mathrm{Fe}_{1-x} \mathrm{~V}_{x}$ alloys with $0.4<x<0.8$ are shown in Fig. 6. They reveal that the linewidth of the Mössbauer spectra is much wider than the natural linewidth of ${ }^{57} \mathrm{Fe}$. These phenomena can be ascribed to a distribution of the electric field gradient, because the amorphous and bcc $\mathrm{Fe}_{1-x} \mathrm{~V}_{x}$ alloys with $x>0.7$ are paramagnetic at $290 \mathrm{~K}$. Since the bcc $\mathrm{Fe}_{0.38} \mathrm{~V}_{0.62}$ alloy is ferromagnetic at $290 \mathrm{~K}$, the observed line broadening is partly ascribed to the hyperfine field. The distribution of the quadrupole splitting, QS, was obtained by an analysis similar to Window's method ${ }^{(16)}$. In order to fit the broad and asymmetric spectrum, a linear relation between the isomer shift and the quadrupole splitting was assumed ${ }^{(6)}$. The distribution curves of QS of the amorphous and crystalline phases are shown in Fig. 7. The shape of the QS distribution curve of the amorphous phase is different from that of the bcc phase and similar to that of the $\sigma$ phase.

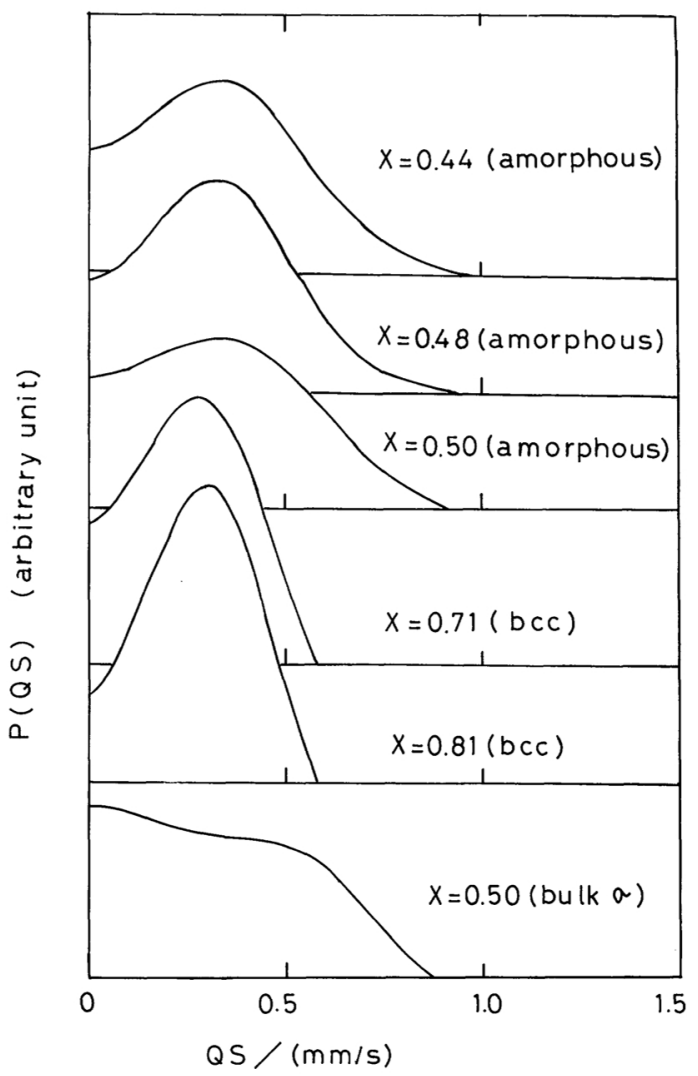

Fig. 7 Distribution of quadrupole splitting, QS, for the $\mathrm{Fe}_{1-x} \mathrm{~V}_{x}$ alloys at $290 \mathrm{~K}$. 


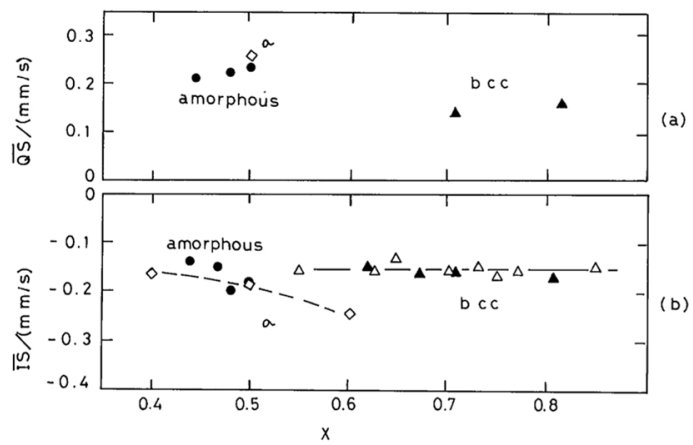

Fig. 8 (a) Average quadrupole splitting. $\overline{\mathrm{QS}}$, for the $\mathrm{Fe}_{1-x} \mathrm{~V}_{x}$ alloys at $290 \mathrm{~K}$. : the vapor quenched amorphous alloys. $\diamond$ : a bulk $\sigma$ alloy. $\boldsymbol{\Delta}$ : the vapor quenched bcc alloys. (b) Average isomer shift, $\overline{\mathrm{IS}}$, for the $\mathrm{Fe}_{1-x} \mathrm{~V}_{x}$ alloys at $290 \mathrm{~K}$. O: the vapor quenched amorphous alloys. $\diamond:$ the bulk $\sigma$ alloys ${ }^{(18)}$. $\mathbf{\Delta}$ : the vapor quenched bcc alloys. $\triangle:$ the bulk bcc alloys ${ }^{(19)}$.

The average values of quadrupole splitting, $\overline{\mathrm{QS}}$, of the $\mathrm{Fe}_{1-x} \mathrm{~V}_{x}$ alloys are shown in Fig. 8(a). $\overline{\mathrm{QS}}$ values of the amorphous alloys are similar to that of the $\sigma$ phase alloy, but are different from those of the bcc alloys. These results suggest that amorphous intertransition metal alloys have a crystal-like short-range structure bearing resemblance to those of the intermetallic conpounds ${ }^{(17)}$.

The average isomer shifts, $\overline{\mathrm{IS}}$, of the $\mathrm{Fe}_{1-x} \mathrm{~V}_{x}$ alloys obtained by the Mössbauer spectra at $290 \mathrm{~K}$ are shown in Fig. 8(b). The isomer shiit of the $\mathrm{Fe}_{1-x} \mathrm{~V}_{x}$ alloys is negative with respect to $\alpha-\mathrm{Fe}$ and insensitive to the alloy concentration. The negative values of the isomer shift are due to electric charge transfer from electropositive $\mathrm{V}$ atoms to electronegative $\mathrm{Fe}$ atoms ${ }^{(18)}$. The values of $\overline{\mathrm{IS}}$ of amorphous alloys are similar to those of the $\sigma$ phase $^{(18)}$ and bcc alloys ${ }^{(19)}$.

\section{Discussion}

\section{Nonequilibrium phase diagram}

The phase boundaries of the bcc and the amorphous phases of the present vapor quenched $\mathrm{Fe}_{1-x} \mathrm{~V}_{x}$ alloys are qualitatively determined by $\mathrm{X}$-ray diffraction measurements. We have the bcc phase for $x<0.4$ and $x>0.5$, while the amorphous phase for $0.45<x<0.5$. Since the bcc phase is ferromagnetic and the amorphous phase paramagnetic, we can esti-

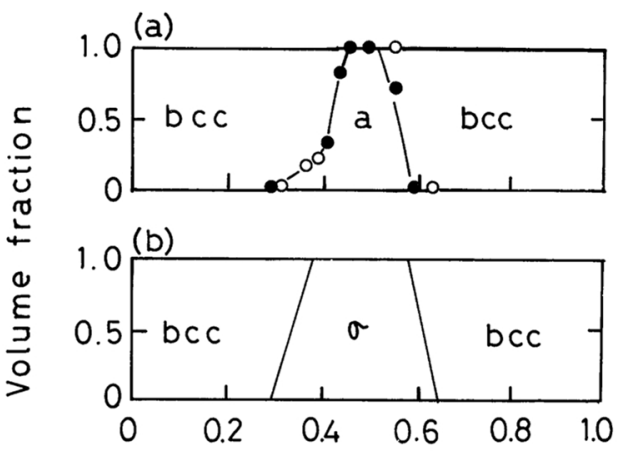

Fig. 9 Volume fraction of the bcc and the amorphous phases in the $\mathrm{Fe}_{1-x} \mathrm{~V}_{x}$ alloys. (a) The present vapor quenched alloys. The open circles are estimated from the magnetization measurements and the closed ones from the Mössbauer effect measurements. (b) The equilibrium state at $570 \mathrm{~K}^{(9)}$.

mate the volume fraction of these phases from the magnetization and Mössbauer effect measurements as shown in Fig. 9.

In some alloys codeposited on a low temperature substrate, the free energy vs composition curve, the $G-x$ diagram, well reflects the structure: The composition at $\Delta G_{\alpha-\sigma}=G_{\alpha}-G_{\sigma}=0$ in the $G$ - $x$ diagram gives the boundary composition of a metastable single phase ${ }^{(20)}$. In the $\mathrm{Fe}-\mathrm{V}$ alloys, the $G-x$ diagram at $350 \mathrm{~K}$ is estimated $^{(21)}$ as shown in Fig. 10. The region of the single bcc phase in the $G$ - $x$ diagram agrees with that of the single bcc phase of vapor quenched alloys. The amorphous phase and the bccamorphous mixed phase are obtained by vapor quenching in the region where the $\sigma$ phase is more stable than the bcc phase in the $G-x$ diagram. This result suggests that the amor-

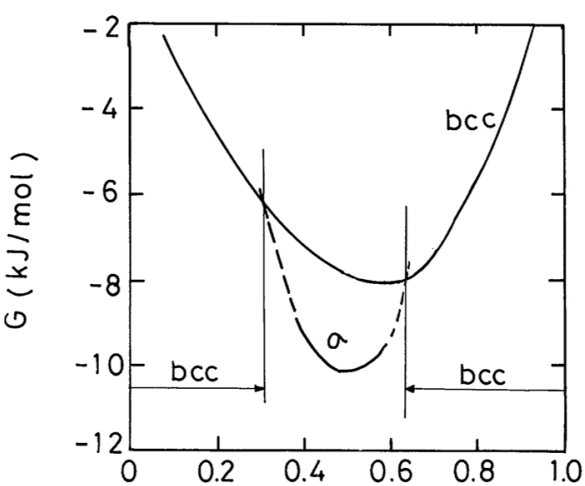

Fig. 10 Free energy, $G$, versus $\mathrm{V}$ concentration, $x$, curves for the bcc and the $\sigma \mathrm{Fe}_{1-x} \mathrm{~V}_{x}$ alloys at $350 \mathrm{~K}^{(21)}$. 
phous phase appears if the formation of a compound with a complicated structure, such as the TCP, is inhibited.

Since many lattice defects and strain are introduced in the vapor quenched $\mathrm{Fe}-\mathrm{V}$ alloys, compositional disorder and dispersion of the interatomic spacing in the TCP phase may give rise to a structure similar to that of an amorphous phase (see Figs. 7 and 8). Since d-electron bondings play an important role in the formation of TCP phase ${ }^{(22)}$, they may also contribute to the amorphous phase formation. The average number of the d-band hole, $\bar{N}_{\mathrm{h}}$, would be a measure for the d-electron bonding ${ }^{(23)}$. In the $\mathrm{Fe}-\mathrm{V}$ system, the $\sigma$ phase is obtained for $\bar{N}_{\mathrm{h}}=3.6-4.3^{(23)}$, and similarly the amorphous phase for $\bar{N}_{\mathrm{h}}=3.9-4.1$.

Another important factor of the amorphous phase formation is the atomic volume mismatch $^{(24)}$. In the $\mathrm{Fe}-\mathrm{V}$ system, atomic radii of $\mathrm{V}$ and $\mathrm{Fe}$ are 0.134 and $0.128 \mathrm{~nm}$, respectively ${ }^{(24)}$. Mader empirically proposed that amorphous phases are often realized for the alloys with the atomic size ratio of the components larger than $1.1^{(1)}$. Although the present $\mathrm{Fe}-\mathrm{V}$ system with the atomic size ratio of 1.05 does not satisfy this condition, we obtain the narrow concentration region of the amorphous phase. On the contrary, in the $\mathrm{Fe}-\mathrm{Cr}$, system, which has also a $\sigma$ phase in the equilibrium state ${ }^{(25)}$ and has the atomic size ratio of $1.02^{(24)}$, an amorphous phase has not been obtained ${ }^{(4)(26)}$. These results support the argument that the stress induced by the atomic size difference in a solid solution leads to the topological instability of the crystalline solid solution, giving rise to an amorphous state ${ }^{(24)}$.

\section{Magnetism of amorphous alloy}

The $\sigma$ phase has five crystallographically non-equivalent lattice sites, I, II, III, IV and V. The I and IV sites are occupied by $\mathrm{Fe}$ atoms, the II sites by V atoms and the III and $\mathrm{V}$ sites by either $\mathrm{Fe}$ or $\mathrm{V}$ atoms. The bulk $\sigma \mathrm{Fe}-$ $\mathrm{V}$ alloys are ferromagnetic and the $\mathrm{Fe}$ atoms in different crystallographic sites carry different magnetic moments. The coordination numbers, CN, of I, III, IV and V sites are 12, 14,12 and 14, respectively ${ }^{(27)}$. These high $\mathrm{CN}$ values give smaller moments in the $\sigma$ phase than that in the bcc phase with a low $\mathrm{CN}$ value of 8. Actually a modified Pauling valence theory gives the magnetic moments at the I, III, IV and V sites as $0,0.7,0.1$ and $0.4 \mu_{\mathrm{B}}$, respectively, for the bulk $\sigma \mathrm{Fe}_{0.52} \mathrm{~V}_{0.48}$ alloys ${ }^{(13)}$. On the basis of the dense random packing of hard sphere structure, the average $\mathrm{CN}$ value of the amorphous state is estimated at $12.6^{(28)}$. These high $\mathrm{CN}$ values of $\mathrm{Fe}$ atoms may result in the reduction of magnetic moments in the amorphous $\mathrm{Fe}-\mathrm{V}$ alloys.

\section{Acknowledgements}

The authors wish to thank Dr. M. Shiga for valuable suggestion in the analysis of Mössbauer spectra. They also thank Mr. T. Unesaki for EPMA (Hitachi X-650) and Mr. K. Kimura for fluorescence X-ray analysis. This work was supported partially by a Grantin-Aid for Developmental Scientific Research (Grant No. 60850132) and Grant-in-Aid for Co-operative Research (No. 61302073) given by the Ministry of Education, Science and Culture of Japan.

\section{REFERENCES}

(1) S. Mader: J. Vac. Sci. Tech., 2 (1965), 35.

(2) K. Sumiyama and Y. Nakamura: J. Magn. Magn. Mater., 35 (1983), 219.

(3) K. Sumiyama and Y. Nakamura: Rapidly Quenched Metals, ed. by S. Steeb and H. Warlimont, Amsterdam, North-Holland, (1985), p. 859.

(4) K. Fukamichi and R. J. Gambino: IEEE Trans. Mag., 17 (1981), 3059.

(5) K. Sumiyama, Y. Hashimoto and Y. Nakamura: Trans. JIM, 24 (1983), 61.

(6) K. Sumiyama, Y. Hashimoto and Y. Nakamura: Trans. JIM, 24 (1983), 66.

(7) K. Sumiyama, H. Ezawa and Y. Nakamura: Phys. Status. Solidi., (a)93 (1986), 81.

(8) K. Sumiyama, H. Ezawa and Y. Nakamura: to be published Acta Metal.

(9) M. Hansen and K. Anderko: Constitution of Binary Alloys, 1st Suppl., New York, McGraw-Hill, (1958), p. 729.

(10) W. B. Pearson: A Handbook of Lattice Spacings and Structures of Metals and Alloys, New York, Pergamon, (1985), p. 663.

(11) A. Kinbara: Sputtering Phenomena, Tokyo, TokyoUniv. Pub., (1984), p. 194 (in Japanese).

(12) M. V. Nevitt and A. T. Aldred: J. Appl. Phys., 34 (1963), 463.

(13) N. Mori and T. Mitsui: J. Phys. Soc. Jpn., 26 (1969), 1087.

(14) J. Hesse and A. Rübartsch: J. Phys. E: Sci. Instrum., 
7 (1974), 526.

(15) M. Shiga and Y. Nakamura: J. Phys. F: Met. Phys., 8 (1978), 177.

(16) B. Window: J. Phys. E: Sci. Instrum, 4 (1971), 401.

(17) R. Wang: Theory of Alloy Phase Formation, ed. by L. H. Bennett, New York, Met. Soc. AIME, (1980), p. 472 .

(18) A. M. van der Kraan, D. B. de Mooij and K. H. J. Buschow: Phys. Status Solidi, (a)88 (1985), 231.

(19) R. S. Preston, D. J. Lam, M. V. Nevitt and D. O. van Ostenburg and C. W. Kimball: Phys. Rev., 149 (1966), 440.

(20) N. Saunders and A. P. Miodownik: Calphad, 9 (1985), 283.

(21) P. J. Spencer and F. H. Putland: J. Iron Steel Inst., 211 (1973), 293.
(22) B. C. Giessen and D. E. Polk: Theory of Alloy Phase Formation, ed. by L. H. Bennett, New York, Met., Soc. AIME, (1980), p. 487.

(23) R. E. Watson and L. H. Bennett: Acta Metal., 32 (1984), 477.

(24) T. Egami and Y. Waseda: J. Non-Crystalline Solids, 64 (1984), 113.

(25) R. P. Elliott: Constitution of Binary Alloys, 1st Suppl., New York, McGraw-Hill, (1965), p. 345.

(26) K. Sumiyama, K. Ohshima and Y. Nakamura: in preparation.

(27) Y. Sumitomo, T. Moriya, H. Ino and F. E. Fujita: J. Phys. Soc. Jpn, 35 (1973), 461.

(28) T. Egami and V. Vitek: Amorphous Materials: Modeling of Structure and Properties, ed. by V. Vitek, New York, Met. Soc. AIME, (1983), p. 127. 